Final version published as: Schwartz PH. Decision and discovery in defining 'disease'. In: Kincaid H, McKitrick J, editors. Establishing medical reality: essays in the metaphysics and epistemology of

biomedical science. Dordrecht: Springer; 2007. p. 47-63. [http://dx.doi.org/10.1007/1-4020-5216-2_5]

\title{
Decision and Discovery in Defining “Disease”
}

\author{
May 17, 2005 \\ Peter H. Schwartz \\ Indiana University Center for Bioethics and \\ Boston Center for the Philosophy and History of Science \\ Forthcoming in Establishing Medical Reality: Essays in the Metaphysics and \\ Epistemology of Biomedical Science, eds. H. Kincaid and J. McKitrick. \\ Dordrecht: Springer.
}

A Case.

Two weeks ago, Mr. Smith caught a cold. His runny nose cleared up in a week, but the cough continued and a few days ago it got worse. Last night he couldn't sleep because he was coughing so much, and for the first time he developed a fever and chills. Today his temperature is $101 \mathrm{~F}$, he is coughing up some brownish sputum, and he feels short of breath. He has pneumonia - confirmed by the physical exam and chest x-ray - and is admitted to the hospital for antibiotics, fluids, and observation.

\section{Introduction:}

The debate over how to analyze the concept of disease has often centered on the question of whether to include a reference to values, in particular the "disvalue" of disease, or whether to avoid such notions. Using Mr. Smith's condition as an example, it is easy to see the attractive aspects of each approach. "Normativists,” such as King ([1954], 1981) and Culver and Gert (1982) emphasize the undesirability of diseases and the harms and limitations they bring, and Mr. Smith’s condition certainly has these features. “Naturalists,” most prominently Christopher Boorse (1977, 1987, 1997), instead require just the presence of biological dysfunction, and again Mr. Smith’s case fits the theory: the infection is now interfering with his lungs' ability to carry out the function of absorbing oxygen and releasing carbon dioxide.

The debate between normativism and naturalism often deteriorates into stalemate, with each side able to point out significant problems with the other. It starts to look as if neither approach can work. In this paper, I argue that the standoff stems from deeply 
questionable assumptions that have been used to formulate the opposing positions and guide the debate. In the end, I propose an alternative set of guidelines that offer a more constructive way to devise and compare theories.

In Section 2, I describe some of the current methodological precepts and provide a way of classifying theories that avoids the classic dichotomy of normativism vs. naturalism. In Sections 3 and 4, I present an overview of the strengths and weaknesses of four of the most important theories of “disease.” In Section 5, I argue against the idea that there is just one correct definition to be discovered and that this definition must be a short list of necessary and sufficient criteria. These assumptions - part of the project of conceptual analysis according to many philosophers - are undermined by psychological research on concept-use and philosophical arguments about meaning.

Once these assumptions have been dropped, the way is open for a more productive approach, which I outline in Sections 5 and 6. In particular, the new framework allows us to give adequate importance to both values and biological dysfunction in defining “disease.”

\section{Classification and Methodology}

\subsection{Classifying Theories:}

The first step in disentangling the debate is to move beyond the terminology of “naturalism” vs. “normativism.” Instead, I will use the term "value-requiring” (VR) for theories that define “disease” using concepts such as “disability,” "harm” or “evil.” Theories relying on such ideas include Lester King’s ([1954], 1981) account, which defines diseases as conditions that are judged by society to be “painful, or disabling” (p. 112). Culver and Gert (1984) similarly invoke values but drop King’s relativist approach, focusing instead on the objective features that make diseases undesirable to many.

For the opposing side, instead of "naturalist," I prefer the more precise "nonvalue-requiring” (non-VR). Christopher Boorse’s $(1977,1987,1997)$ theory, for instance, is non-VR, since he includes no criterion involving the value or disvalue of a 
condition. Instead, his theory requires just the presence of biological dysfunction, and thus I will call the account “dysfunction-requiring” (DR). King ([1954], 1981) and Culver and Gert (1984), in contrast, do not refer to the presence of dysfunction, so their accounts are "non-dysfunction-requiring” (non-DR). The benefit of this more precise terminology becomes apparent when we consider Jerome Wakefield's theory, which requires both the presence of dysfunction and the tendency to cause harm. His theory is thus both dysfunction-requiring and value-requiring (DR and VR). The advent of a theory such as Wakefield's helps illustrate how the normativist/ naturalist divide is starting to break down (see also Richman 2004).

I’ll focus on these four theories: King (\{1954], 1981), Culver and Gert (1982), Boorse (1977, 1987, 1997), and Wakefield (1992a, b, 1999a, b). Their strict definitions are listed in Table 1. They can be classified by using a 2x2 grid (Table 2), with the horizontal axis divided between dysfunction-requiring (DR) and non-dysfunctionrequiring (non-DR), and the vertical axis divided between value-requiring (VR) and nonvalue-requiring (non-VR). The theories thus fall in three of the four squares of this grid, and I will take the strengths and weaknesses of each as representative of these general approaches.

\section{2 "Disease" and Methodology:}

All these accounts offer their definitions as necessary and sufficient criteria for a condition's being a disease. In so doing, they reflect what has been called the "classical view” of concepts, where concepts are represented by a short list of necessary and/or sufficient criteria for their application (Ramsey 1992). As mentioned above, the classical view is generally assumed in analytic philosophy, as can be seen in the conceptual analyses of concepts from “cause” in metaphysics, to “justification” in epistemology, to “justice” in moral theory.

The four theories to be considered here vary in their choice of terms - King and Boorse use “disease,” Culver and Gert use “malady,” and Wakefield uses “disorder” but all treat the relevant notion as encompassing all pathological conditions. As Boorse writes, in many contexts the term "disease” applies to only a subset of conditions he is 
interested in, leaving out such things as injuries and malformations. But he says that he means "disease" in a broad sense, taken as analogous to "a pathologist's concept of disease" (1997, p. 11). Culver and Gert, likewise, say that they are not interested in the "arbitrary” distinctions between concepts such as "disease," "illness,” "injury,” "defect,” and "injury," aiming instead to encompass them all with their term "malady" (1982, p. 65). And while Wakefield is mostly concerned with the concept of "mental disorder," he writes that he is interested in a notion that applies to physical disorders as well (1999a, p. 376). I'll use the term "disease" for the concept in question, while deferring to the terms used by the individual theories in appropriate places.

Each definition is generally supported by arguments that it does a better job than the others at classifying conditions as diseased or healthy. The arguments thus depend on which examples are chosen and what criteria are used to determine the correct classification. Unfortunately, some of the key debates in the naturalist vs. normativist debate have involved completely imaginary cases and unsupported intuitions about what doctors or others would or should say about them. Many projects of conceptual analysis in analytic philosophy rely on such imaginary cases and armchair intuitions - think of the convoluted and occasionally physically-impossible cases used in the debate over causation or personal identity - and while I do believe such cases can be useful, they must be used with care. I will focus on arguments where historical or contemporary examples are used and where claims about classification are supported by good arguments or actual practice.

\section{Values and Disease}

\subsection{Culturally-Relative Values:}

Lester King presented one prominent value-requiring (VR) definition of "disease" 50 years ago, writing,

"Disease is the aggregate of those conditions which, judged by the prevailing culture, are deemed painful, or disabling, and which, at the same time, deviate 
from either the statistical norm or from some idealized status." ([1954], 1981, p. 112).

The relevant values here are defined as culturally relative, based on what the "prevailing culture” believes, and there's much to recommend this sensitivity to cultural judgments. Historians and sociologists of medicine often focus on moral beliefs and value judgments in analyzing how societies decide which conditions will count as normal and abnormal. Such analyses often uncover how value judgments in this area interact with other beliefs, as well as with political and economic forces (c.f. Rosenberg 1992).

For VR theories, though, adopting a relativist notion of value also carries negative consequences. In particular, it makes it difficult to explain how societies can be mistaken about what is a disease. Masturbation was considered a disease in 19th century America (Engelhardt 1974), as was the desire of slaves to escape, labeled “drapetomania.” It's not just that we have now stopped counting masturbation or a desire for freedom as diseases, it's that we believe that ever seeing them in this way was deeply mistaken. Similarly, some conditions previously considered normal and even desirable are now judged to be pathological. Take, for instance, the traditional Chinese practice of foot-binding, which often left its subjects unable to walk. Classifying masturbation as a disease or deformed feet as normal are mistakes, like believing that the world is flat or that spirits cause hurricanes.

Relativists can respond in two ways. They might articulate and defend a wideranging relativism, where even the shape of the earth is a relative matter. More commonly, they will distinguish between such "objective” facts as the shape of the earth and the value-laden judgments concerning states of the body. The most nuanced commentators pursue a sort of middle way. Charles Rosenberg, for instance, rejects the idea that societies "construct" disease, instead arguing that they somehow "frame" it and “negotiate” its bounds (c.f. Rosenberg 1992). I won’t consider the realism/ relativism debate in this area any further here, having at least pointed out the challenges facing a truly relativist approach.

\subsection{Objective Values:}


Other VR accounts avoid relativism by defining the relevant value-laden notions in a non-relativist way. Culver and Gert's theory is representative of this approach. They choose the term "malady" for their target notion and define it as follows:

"A person has a malady if and only if he has a condition, other than his rational beliefs and desires, such that he is suffering, or at increased risk of suffering, an evil (death, pain, disability, loss of freedom or opportunity, or loss of pleasure) in the absence of a distinct sustaining cause." (Culver and Gert, 1982, p. 81)

Here the relevant value-concept, "suffering ... an evil," is precisely defined in terms of a list of possible events - “death, pain, disability, loss of freedom or opportunity, or loss of pleasure." According to their theory, if masturbation or desiring to escape slavery does not actually increase a person's risk of experiencing death, pain, etc., then these conditions are not maladies, no matter what society believes. This avoids the most serious challenge facing the relativist version of VR theories.

That said, Culver and Gert's criterion still allows too many conditions to count as diseases. In a society with certain value beliefs, it may very well be objectively true that a slave’s having a deep desire to escape does carry significant risk of death, pain, etc. In a society with certain beliefs, again, masturbating or rejecting foot-binding will similarly carry real costs in terms of freedom, opportunity, or pleasure. Thus, Culver and Gert really just lengthen the path from societal beliefs to disease-status by one step: the value judgments now determine what counts as disease by way of their influence on people's lives. Similarly, it seems that in a bigoted society having dark skin or appearing to be a member of a certain ethnic group might count as a malady.

Critics have also pointed out that Culver and Gert's theory counts pregnancy as a malady because of the associated disability and the risk of complications and even death. Culver and Gert explicitly accept this conclusion (Gert et al. 1997, p. 126), and this suggests that the concept of malady may differ significantly from the concept of disease that is actually at work in biology or medicine. From a biological point of view, of course, pregnancy and the process of reproduction is a normal state of the organism, no matter how risky. And although medical professionals treat women who are pregnant and Defining Disease p. 6

Peter Schwartz 6-16-05

Draft Copy: Not for Circulation or Citation without Author's Approval 
in labor, it's the conditions that may arise in these settings that count as pathological, rather than the pregnancy itself. Health professionals providing contraception and carrying out abortions generally do not believe that pregnancy is a pathological condition.

These problems for Culver and Gert stem from their making harm a sufficient criterion for disease, and other serious problems stem from its being a necessary criterion. For example, a disease may actually benefit an individual who has it, as when flat feet keep a person out of the army, or when an infection with cowpox confers resistance during a smallpox epidemic (Boorse 1977, pp. 544-5). More fanciful examples are possible, such as a case where a researcher in a totalitarian country saves a person's life to study his mild eczema (following Boorse 1997, p. 88). In these situations the conditions are still diseases, it seems, just ones that happen to be beneficial.

A VR account could respond in a couple of ways, for example by emphasizing that all these conditions carry some harm that is being counterbalanced by certain benefits. But allowing a condition to count as a disease just as long as it carries some harm - independent of the net outcome - would water the condition down to triviality, since all conditions carry at least some harm. Winning the lottery, for instance, carries the "harm" of wondering what to do with the money and who is your real friend, etc. Another approach for a VR account responding to these problems would be to define some environments as normal and others as abnormal, requiring only that the condition causes net harm in normal environments. This way forward, however, depends on a concept of "normal environment" that appears as difficult to define as the notion of disease was in the first place.

\section{Dysfunction and Disease:}

\subsection{Boorse's Account:}

Partially in response to the problems involved with making values necessary or sufficient to a condition's being a disease, Boorse $(1977,1987,1997)$ proposes entirely eliminating value judgments from the definition. Instead, he focuses exclusively on 
whether there is biological dysfunction present. He states his "Biostatistical Theory" (BST) most recently as follows:

1. The reference class is a natural class of organisms of uniform functional design; specifically, an age group of a sex of a species.

2. A normal function of a part or process within members of the reference class is a statistically typical contribution by it to their individual survival and reproduction.

3. A disease is a type of internal state which is either an impairment of normal functional ability, i.e. a reduction of one or more functional abilities below typical efficiency, or a limitation on functional ability caused by environmental agents.

4. Health is the absence of disease. (1997, pp. 7-8)

Each of the first three premises is central to applying his dysfunction-requirement. First, organisms are compared just with other individuals of similar age and same gender, i.e. members of the same "reference class" defined in Premise 1. This is because levels of functioning that count as normal vary over age and between the genders in crucial ways. Human children younger than six months old can't walk and newly hatched chicks can’t fly, but their legs and wings (respectively) do not count as dysfunctional. In mammals, the female breast has the function of producing milk at certain times, while the male breast never does. Note that even value-requiring accounts may need to rely on some form of "reference classes," for example to explain when an individual has an “increased risk” of suffering an evil (Culver and Gert 1982, quoted above).

Second, a trait's function is defined (in Premise 2) as its "statistically typical contribution” to survival and reproduction in individuals with that trait. Thus the eyes have the function of allowing sight, the heart has the function of pumping blood, and the lungs have the function of absorbing oxygen and releasing carbon dioxide. Philosophers differ about the best definition of "function," with some linking the notion to the evolutionary history of the relevant trait (c.f. Neander 1991). I won't address the debate between these accounts and Boorse's here, beyond noting that both work equally well in classifying Mr. Smith’s case of pneumonia as disease, since both assign the lungs the 
same function. The lungs contribute to survival and reproduction by absorbing oxygen and releasing carbon dioxide, and they clearly arose under selection at least partly for carrying out these roles.

Third, the idea of a condition's being an “impairment of normal functional ability,” in Premise 3, is meant to be a roughly statistical distinction. If a trait's level of functioning falls in the lowest few percentile for people of the same gender and age, then it counts as dysfunction. In this way, the definition is meant to avoid any of the notions of disability or harm that value-requiring accounts rely upon. Mr. Smith’s pneumonia counts as a disease, according to this account, since his lung function has decreased significantly. His lungs are carrying out enough gas exchange to keep him alive but are functioning at levels that are statistically unusual for men of his age. And it is this diminished functioning that makes Mr. Smith’s case of pneumonia a disease, not the suffering or risks that it imposes.

\subsection{Challenges to Boorse:}

Boorse's theory has been the most prominent non-value-requiring account and thus has attracted a great deal of criticism, which Boorse has recently addressed in a monograph-length paper (1997). Perhaps the most serious attacks on the account focus on the claim that dysfunction is a sufficient condition for disease. For example, if there is some part of the human brain that produces sexual attraction for members of the opposite sex, then this part would have the function of so doing, since this role is its statistically typical contribution to reproduction (as in Premise 2). But then it follows that if this part of a person's brain fails to produce such attraction in a small percentage of the population, resulting in homosexuality, this condition would count as a case of disease, by Premise 3. And this clearly contradicts the opinion of the medical profession and most people in the United States.

Boorse has bitten the bullet on this, accepting that it may turn out that his account makes homosexuality a disease, and he defends the theory by pointing out that the fact that a condition is a disease carries no inevitable implications about whether it should be treated by doctors (1997, pp. 11-13). Infertility would also be a disease, according to his 
theory, but a person who preferred to remain childless might understandably reject treatment as well. As mentioned in Section 2, Boorse says that he's interested in defining the theoretical notion of disease at work in medical science, leaving decisions about whether to intervene to people's free will. And these decisions, he accepts, will involve the application of values. He proposes that there might even be a different concept, which he calls "therapeutic abnormality," that should be defined by adding a valuerequirement to his definition of “disease” (1997, pp. 12-13).

Other challenges to Boorse's account object to the claim that dysfunction is a necessary criterion for a condition's being a disease. For example, it may be the female orgasm makes no specific contribution to survival or reproduction, and thus the mechanisms that bring it about have no biological function. But at the same time, a woman's inability to orgasm may be a serious problem for her, and one that physicians should treat as a disease (Reznek 1987, p. 131). Again, Boorse responds by appealing to the distinction between a condition's disease-status and questions about whether medical professionals should diagnose and treat it if possible. Even if anorgasmia is not a disease, he writes, the suffering it causes may be a sufficient reason for health professionals to attempt to alleviate it. While the treatment of disease makes up a central part of medical practice, he writes, there may well be other conditions, which are not diseases, that health professionals have the skill and training to treat (Boorse 1997, pp. 90-99). Doctors have long been involved in inducing sterility and fixing ugly noses, but they do so without claiming that fertility or ugliness are diseases. The cost of this approach, though, is to separate Boorse’s favored notion of “disease” from any clear conclusions about what medical professionals should do, and this risks making his definition look somewhat irrelevant to medical practice.

Both these problems for Boorse's theory - classifying homosexuality as a disease and anorgasmia as healthy - stem from the account's focus on the way that a trait affects just survival and reproduction. So even though homosexuality may be consistent with, or conducive of, pleasurable, loving lives, the condition counts as a disease because of its interference with certain ways of reproducing. And even though sexual satisfaction may be essential to many people’s happiness, a condition like anorgasmia only counts as a 
disease if it interferes with reproduction. The focus on reproduction looks like it leaves out too much, i.e., all the other crucial goals and goods of human life. Of course, those are exactly the goods that VR accounts like King's and Culver and Gert's attempt to include, and we saw the problems with including so much. It starts to look as if the attempt to define disease faces a sort of dilemma: either include too much or too little of normal human goals.

\subsection{Wakefield's Account}

Jerome Wakefield (1992a, b, 1999a, b) attempts to answer some of these problems by combining a dysfunction-requirement with a value-requirement. He states his “Harmful Dysfunction” account as follows:

A condition is a disorder if and only if (a) the condition causes some harm or deprivation of benefit to the person as judged by the standards of the person's culture (the value criterion), and (b) the condition results from the inability of some internal mechanism to perform its natural function, wherein a natural function is an effect that is part of the evolutionary explanation of the existence and structure of the mechanism (the explanatory criterion). (1992a, p. 384)

The theory thus avoids Boorse's apparent misclassification of homosexuality: even if homosexuality results from a biological dysfunction of some part of the brain, it is not a disease if it doesn't impose harm. Since the presence of dysfunction is necessary but not sufficient in Wakefield's account, it can avoid some of the problems facing Boorse.

That said, Wakefield's theory still has difficulties that stem from dysfunction's being a necessary condition: female anorgasmia will still not count as disease if orgasm has no function in women. And there are cases that do not involve sexuality or reproduction that can be used to attack the dysfunction-requirement in similar ways. (These examples challenge Boorse's account just as much, of course, since he treats dysfunction as necessary as well as sufficient.)

Consider Mr. Smith's case of pneumonia again, slightly modifying the facts so that he has the bad cough and fever but no problem with his breathing. While he has 
pneumonia, since he has fluid in some alveoli, he has preserved lung function. Assume that his doctor properly diagnoses and treats him, and he gets better. But then it's not clear why his case of pneumonia counted as disease, according to Boorse's or Wakefield's theories, since it's not clear where the dysfunction was. The lungs were able to carry out their function of gas exchange, and the immune system carried out its function of fighting the infection. Although the cough and fever were unpleasant, they were also important components of the body's response to the microbe. People without a good cough are at greatly increased risk of serious respiratory infections, and fever may well be an adapted response that weakens the bacteria's ability to reproduce.

So although a serious case of pneumonia involves biological dysfunction, it's not clear that a mild case does too. And we can come up with many cases like this. Although measles is a disease, in most cases there is no clear dysfunction.

Boorse responds to similar concerns by pointing to what he calls dysfunction at the cellular level: even in a cold, he writes, cells in the mucosa are dying. Responding to some critics, he even claims that the death of a single cell means that disease is present (1997, pp. 50-1). But this makes the necessary condition so easy to satisfy that it verges on triviality. During menstruation, after all, there is massive cell death as the uterine lining is shed. And during the third-trimester of pregnancy the large uterus interferes with the normal function of the bladder storing urine and of the veins carrying blood back from the legs. But menstruation and pregnancy are normal, healthy conditions.

Wakefield's "value criterion” blocks some of the attacks on Boorse's theory based on such cases, as described above for homosexuality. But since the value criterion requires that there is "some harm or deprivation of benefit to the person, as judged by the standards of the person's culture," the theory faces some of the same counterexamples that confronted King ([1954], 1981). If a culture values eczema or bound-feet, then these conditions will not count as pathological. Finally, even without the cultural relativity, making harm a necessary requirement opens the theory to counterexamples involving diseases that benefit their victims, such as flat feet keeping a young man out of the army or cowpox conferring immunity during a smallpox epidemic. 
At this point, it starts to feel like attempting to craft a definition of disease is like the scene in the movie Fantasia where the sorcerer's apprentice is trying to eliminate the magical brooms: crush one, and two spring up. Or, it's like a nightmare version of Hans Brinker: plug one hole in the dyke, and immediately another one opens up. At this point, it makes sense to reconsider the project overall.

\section{Conceptual Analysis and an Alternative Methodology.}

\subsection{Conceptual Analysis and Criteria of Application:}

All these theorists, and basically all contributors to the debate, have assumed that the goal is to provide an adequate conceptual analysis of disease. Current accounts of the project of conceptual analysis generally assume that the target is describing either (i) the meaning of the concept in question or (ii) the "criteria of application" in the minds of competent speakers (c.f. Neander 1991). And, as mentioned above, both these approaches adopt the “classical view” of concepts, where concepts are represented by short lists of individually necessary and jointly sufficient conditions. Here I will argue that conceptual analysis, formulated in keeping with (i) or (ii), and the classical view of concepts associated with this project, is deeply problematic.

The claim to be uncovering a "criteria” at work in the minds of speakers runs afoul of psychological research on the use of concepts. First of all, psychologists have undermined the classical view of concepts by showing that objects are usually classified based on characteristics that are not strictly necessary (Ramsey 1992, Rosch and Mervis 1975). For example, it counts towards an object's being a chair that it has four legs, but some chairs don't have legs at all. The fact that an animal flies counts towards its being a bird, but some animals are birds even though they don’t fly.

Second, research has shown that the way speakers classify objects is a matter of degree, rather than an all-or-nothing affair, as the classical view implies (Ramsey 1992, p. 63). Items can count as better or worse examples of a given concept. For instance, speakers see a kitchen-table chair as a better example of a chair than the five-foot-high chair that a tennis referee sits in, and speakers treat falcons and hawks as better examples 
of birds than ostriches. This ordering of items is reflected by many different measures: more central examples of a concept are learned more easily, come to mind more quickly, and influence thought more powerfully (Ramsey 1992, p. 63). In addition, context can change which properties count as more important for classifying an object and for determining whether it is a good example of the concept in question (Ibid.).

These findings have led psychologists to adopt models that resemble Wittgenstein's “family resemblance” (1953) picture of concepts rather than the classical view. According to some such models, for instance, objects are classified based on whether they resemble a prototype in certain ways, measured according to some "similarity metric." In one quantitative model, concepts are represented by lists of properties, each of which has a point value: items that accrue enough points count as falling under the concept. In this way, different objects falling under the same concept may share few (or perhaps no) important properties. And it's relatively easy to make sense of which items count as a "good example": a higher point total means a better example.

Neander (1991) describes her project of conceptual analysis - in this case, focused on the biological concept of function - as aiming to uncover the "criteria of application" that are at work in the minds of biologists. And she argues that psychological research that undermines the classical view, described above, does not weaken her project since "function" is a theoretical term used by experts, rather than one used by general speakers in common language. "Function," she claims, is like "water" for chemists, which has necessary and sufficient conditions for its application (1991, pp. 172-3).

I've argued elsewhere (2004, p. 146) that the analogy is not apt for "function," and it certainly isn't for "disease" either. Although it is true in both cases that the term is used by "experts"- biologists and doctors, respectively - there is no commonly accepted technical definition that these experts share, or acquire in training, as in the case of "water." The absence of any technical meaning for "disease" can be seen in the heated disagreements among experts over whether infertility, short-stature, or obesity are truly 
diseases (Konner 1999). In fact, it is the lack of such a commonly accepted meaning for "disease" and for "function" that attracts the interest of philosophers to these areas.

Another problem for conceptual analysis, independent of the findings that undermine the classical view of concepts, is the fact that people may rely on widely varying criteria when applying a given concept. For example, it is likely that different people will use the term "disease" in different ways and that even a single person will use it in different ways at different times. There may be significant disparity between the use of the term by laypeople and by doctors, as well as among the various subfields of medicine. There's really no way to characterize the range of variation unless psychologists were to study people's use of the term in various real and imagined situations. And this is a project very different from the sort of analysis that philosophers generally employ. Either they've misdescribed the project, or they've chosen an unsuitable method for pursuing it.

\subsection{Conceptual Analysis and Meaning:}

A different, and more common, defense of conceptual analysis avoids any claim concerning people's use of the term in question, and instead aims to uncover the term's or the concept's meaning. According to this picture, each concept has a meaning that lies behind the actual usage and understanding of the concept and which guides its use in some way. Competent speakers may apply the concept by using similarity to prototypes or other methods, but the meaning, it is argued, is understood by all and can be discerned by careful philosophical study.

Although this picture is attractive in some ways, it assumes a notion of meaning that has been seriously undermined by analytic philosophy of language. One way to articulate the problem is as follows. Assume for a minute that such a meaning of "disease" exists, and that we are assured that Mr. Smith has a disease. Then all other claims about Mr. Smith's condition can be classified as analytic, i.e. following from the meaning of "disease," or as synthetic, i.e. requiring more information. Which statements are analytic and which are synthetic depends on which definition of "disease" is correct. For instance, if Culver and Gert's definition is right, then it follows analytically that Mr. 
Smith is at increased risk of an evil (as they define that term); it follows just synthetically that his lungs are dysfunctioning. In contrast, if Boorse's account of "disease” is correct, then the statement that his lungs are dysfunctioning follows analytically and the statement that he's at increased risk of an "evil" follows synthetically.

The problem with these conclusions is that good arguments have undermined the claim that there is a fact of the matter which statements are analytic and which are synthetic, in relation to a given proposition. Quine (1953) highlights the difficulty determining whether "he is unmarried" follows analytically or synthetically from "he is a bachelor," and explains why, given certain assumptions, such a distinction cannot be a factual matter (1961). Putnam (1962) uses examples from history of science to show how problematic it is to determine which statements of science are analytic, in the sense of following just from the meaning of the terms, and which are synthetic. For example, he points out, the equation "e $=1 / 2 \mathrm{mv}^{2}$ " was initially a definition of kinetic energy and thus analytic. But later, arguments for relativity theory disproved the equation, suggesting that at some point it had become a synthetic claim. It is not "happy," Putnam says, to force all scientific statements into the categories of analytic or synthetic.

If this is so, then it also can't be the case that the terms of science have strict definitions, the sort that might be uncovered by conceptual analysis. I will not delve into the arguments for or against the analytic/ synthetic distinction further here, beyond pointing out that the important arguments attacking it undermine the idea that the analysis of "disease" should be seen as a search for the meaning of that concept.

\subsection{Conceptual Analysis, Natural Kinds, and Stipulation:}

In order to avoid such problems, some philosophers have suggested that the analysis of concepts should be seen as a search for necessary and sufficient conditions determining the extension. This picture seems to fit discoveries concerning natural kind terms, such as the discovery that water is just $\mathrm{H}_{2} \mathrm{O}$. Millikan (1989) presents her definition of "function" in this light, not as a claim about meaning, but as a characterization of a phenomenon that biologists have investigated. And she adamantly 
denies that her "theoretical definition" has anything to do with an attempt to uncover the meaning of the term or the criteria used by speakers for applying it.

There's much to be said for this view, although its overall attractiveness depends on what account of natural kind terms it is paired with. On some views, the criteria for membership in the extension of a natural kind term is discovered, as humans discovered that gold is all and only atoms with atomic number 79 and water is all and only molecules of $\mathrm{H}_{2} \mathrm{O}$. On this view, the terms "gold” and "water" always had these extensions, even if nobody knew it (Putnam 1975). Opposing this view is the idea that the boundaries in such cases are chosen, in some sense, rather than discovered (Donnellan 1983, Dupre 1993) ${ }^{1}$.

But whatever conclusion is drawn for "gold" or "water," the case for "disease” most likely best fits the latter model. As scientists have acquired better and better understanding of diseases and their causes, they find not a unifying microstructure, as for gold or water, but variation. While many have sought an essence that all and only diseases share, this quest has been blocked at every step by variability and heterogeneity. Any definition that would draw a sharp line through all conditions, determining for each whether it is a disease or not, looks like the imposition of a decision, rather than the application of a discovery.

This means that adopting any precise account will impose at least some changes on our currently non-reflective and relatively unprincipled way of distinguishing disease from health. Choosing a definition will partly involve deciding which changes from current practice are acceptable. This picture of the philosophical examination of the concept of disease resembles a general approach put forward in analytic philosophy by Carnap (1950, pp. 3-8) and Quine (1961, pp. 257-262)². As Quine writes,

\footnotetext{
1 See Ebbs 200, 2002 for a more complete analysis of this question.

2 Gary Ebbs has recently utilized a similar project in philosophy of language (2002), and he first recommended the Quinean approach to me (personal communication). Ramsey $(1992,69)$ also mentions Carnap’s (1950) approach as a possible response to problems with conceptual analysis. See also Schwartz (2004), and Lewens (2004). 
We do not claim synonymy. We do not claim to make clear and explicit what the users of the unclear expression had unconsciously in mind all along. We do not expose hidden meanings,... We fix on the particular functions of the unclear expression that make it worth troubling about, and then devise a substitute, clear and couched in terms to our liking, that fills those functions. (pp. 258-9)

While Quine’s overall approach to language and meaning, with its strict behaviorism, does not fit current analytic philosophy, this recommendation is in keeping with the pragmatic bent of much current thought. In a previous paper (2004), concerning the analysis of the concept of "function,” I've recommended this approach and called it “Philosophical Explication” (pp. 143-5).

\section{Philosophical Explication of "Disease"}

I believe that the philosophical study of "disease” should similarly be framed as a project of philosophical explication, and thus should give up the attempt to discover the concept's true meaning, its criteria of application, or the boundaries of its extension. Instead, accounts should be seen as proposed new definitions, or groups of new definitions, that can do much of the same work that was done by the vaguely defined concept of disease in the past. Any definition will impose at least some changes and carry at least some counter-intuitive consequences for usage. Imposing such precision may also well mean that no single concept can be relied upon in all situations; there may need to be different definitions for different contexts.

From this perspective, the long debate between normativism and naturalism including the discussion of the four theories described above - serves a crucial role by displaying the advantages and disadvantages of the various approaches. Choosing an account becomes not so much a hunch about which theory is correct but instead a choice of which theory to clarify and apply.

One reasonable question at this point is to ask whether definitions should be stated as necessary and sufficient criteria, as in the classical view, or instead in keeping with some other model, such as the psychologists’ prototypes and similarity metrics. The 
attraction of the classical view is the possibility of providing clear answers in debates over which conditions are really diseases. Many philosophers and physicians attempt to define "disease" partly as a way to decide how to classify and whether to treat borderline or complex conditions such as obesity, infertility, or short stature (Konner 1999, Schwartz 2001). On the other hand, once we see our definitions as decisions rather than discoveries, this motivation for defining “disease” with necessary and sufficient conditions is greatly diminished. Using prototypes and similarity metrics has the advantage of being more in keeping with the actual use of "disease" and other concepts.

In conclusion, I would like to return to the dysfunction-requiring approach, to evaluate how well it does if we adopt philosophical explication as our project and use prototypes and similarity metrics to represent the concept. In particular, the DR approach does a particularly good job making sense of prototypical cases of disease and distinguishing them from healthy, undesirable conditions. Start with the following three types of prototypical diseases ${ }^{3}$ :

1) Infection: severe bacterial infections such as pneumonia, sepsis (infection in the blood), or dysentery (infectious diarrhea);

2) Injury: broken bones or other sorts of wounds (e.g. puncture wounds or lacerations);

3) Organ failure: kidney or liver failure, blindness, or deafness.

I believe that within each category, the most prototypical cases are the most serious ones, i.e. where there is a raging infection, a serious injury, or complete organ failure. And in all these cases, there is clear biological dysfunction as well. In (1) there is dysfunction of the lungs, the GI tract, etc. In (2) there is failure of the broken bone to provide support or of the lacerated skin to keep out infection. In (3) there is dysfunction of the kidney, liver, eyes, or ears.

These cases will also be classified as disease by a value-requiring, non-DR, approach, since each carries pain, loss of freedom, risk of death, etc. But these

\footnotetext{
${ }^{3}$ Of course, I make no claim that these are all the prototypes or that equally good but incompatible ones could be chosen.

Defining Disease

p. 19

Peter Schwartz 6-16-05
}

Draft Copy: Not for Circulation or Citation without Author's Approval 
undesirable outcomes do not distinguish the conditions clearly from other undesirable but healthy conditions. It may be worse to be impoverished or illiterate than to have kidney failure, for example, given the availability of dialysis. Even being deaf may not impose as much disability or loss of freedom as does being unemployed or being swindled out of one's life savings. So if we focus on prototypical cases, which I think makes sense to do as a first step, I believe that a DR criterion works better than a VR one.

This approach still carries the unattractive implication that homosexuality may count as a disease, and this does not fit my intuitions or those of most of the current medical and psychiatric world. The inclusion of a harm requirement, as in Wakefield's account, answers that problem, but such a requirement bring problems of its own, as described above. It may be that this is a place where a sharpened definition of "disease" carries an unavoidable counter-intuitive consequence.

The DR account also faces serious challenges as it moves from severe diseases to less serious ones. As mentioned above, minor injuries and early infections do not clearly involve any biological dysfunction, such as in the case of early pneumonia or most cases of the measles (Section 4.3). A DR account of the sort I'm sketching might respond to propounding some sort of similarity-metric to link such conditions to the more serious ones. Measles and other viral infections, and bronchitis or early pneumonia, are all processes that carry certain risks of dysfunction, and thus risks of causing prototypical disease. But note that it's not just the risk of the future dysfunction but the presence of a process that might cause such problems. Without requiring that such a process is present, then poverty or old age will also count as disease, since they carry risk as well. Thus this approach faces the serious challenge of articulating a tenable notion of "same process."

Consider another condition that does not clearly involve dysfunction: a small kidney stone that results in a good deal of pain and a small amount of blood in the urine, but where the stone is eventually passed with no resulting dysfunction. Given the high prevalence of people who will have a kidney-stone in their lifetime, the formation of a stone doesn't necessarily indicate dysfunction of the kidney. But still, some stones do lead to serious dysfunction of the kidney, and a person who has had one is at risk of 
having others. Once again the idea of there being a process present that carries risk of dysfunction may be a necessary part of classification in this area.

I believe that there is promise here for an interesting and fruitful account of disease. At the same time, there may be equally promising accounts based on a valuerequirement, perhaps also stated in terms of prototypes and similarity metrics. Once we accept that the project of defining "disease" is a constructive one, and that definitions do not have to be stated according to the classical view, there is room for many approaches. Where there was once stalemate, let there be variability and free choice.

\section{References.}

Boorse, Christopher (1977), "Health as a Theoretical Concept,” Philosophy of Science 44: 542-573.

(1987), “Concepts of Health,” in D. Van De Veer and T. Regan, eds., $\underline{\text { Health }}$

Care Ethics: An Introduction. Philadelphia, PA: Temple UP: 359-393.

(1997), “A Rebuttal on Health,” in J.M. Humber and R.F. Almeder, eds., What is

Disease? Totowa, NJ: Humana Press. pp. 1-134.

-------- (2002), “A Rebuttal on Functions,” in Ariew et al. (2002).

Carnap, Rudolf (1950), Logical Foundations of Probability, $2^{\text {nd }}$ ed. Chicago: Chicago Univ. Press.

Culver, Charles and Bernard Gert (1982), Philosophy in Medicine: Conceptual and

Ethical Issues in Medicine and Psychiatry. New York: Oxford Univ. Press.

Daniels, Norman (1985), Just Health Care. Cambridge: Cambridge Univ. Press.

DePaul, Michael R. and William Ramsey (eds.) (1998), Rethinking Intuition. Lanham:

Rowman and Littlefield Publishers, Inc.

Donnellan, Keith S. (1983), "Kripke and Putnam on Natural Kind Terms," in C. Ginet and S. Shoemaker (eds.), in Knowledge and Mind. Oxford: Oxford Univ. Press, 84104.

Dupre, John (1993), The Disorder of Things: Metaphysical Foundations of the Disunity of Science. Cambridge, MA: Harvard University Press.

Ebbs, Gary. (2000), “The Very Idea of Sameness of Extension Over Time,” American 
Philosophical Quarterly 37: 245-268.

(2002), “Learning from Others”, Nous 36: 525-549.

Engelhardt, H. Tristam, Jr. (1974), “The Disease of Masturbation: Values and the Concept of Disease,” Bulletin of the History of Medicine 48 (2): 234-248.

Gert, Bernard; Culver, Charles M., and K. Danner Clouser (1997), Bioethics: A Return to Fundamentals. New York: Oxford Univ. Press.

Goosens, W. (1980), “Values, Health, and Medicine,” Philosophy of Science 47: 100115.

King, Lester ([1954], 1981), “What is Disease?” In Concepts of Health and Disease: Interdisciplinary Perspectives, ed. A. L. Caplan, H. T. Engelhardt, and J. J. McCartney. Reading, MA: Addison-Wesley Publishing Company. pp. 107-118. Konner, Melvin J. (1999), “One Pill Makes You Larger: The Ethics of Enhancement,” American Prospect 42: 55-60.

Lewens, Tim (2004), Organisms and Artifacts: Design in Nature and Elsewhere. Cambridge, MA: MIT Press.

Millikan, Ruth Garrett (1989), “In Defense of Proper Functions,” Philosophy of Science 56: $288-302$.

Neander, Karen (1991), “Functions as Selected Effects: The Conceptual Analyst’s Defense,” Philosophy of Science 58: 168-184.

Putnam, Hilary (1962), “The Analytic and the Synthetic,” in H. Feigl and G. Maxwell (eds.), Minnesota Studies in the Philosophy of Science, Vol. 3. Minneapolis: Univ. of Minnesota Press.

--------- (1975), “The Meaning of “Meaning',” in Mind, Language and Reality:

Philosophical Papers, Volume Two. Cambridge: Cambridge Univ. Press, 215-271.

Quine, W.V.O. (1953), “Two Dogmas of Empiricism,” in From a Logical Point of View. Cambridge, MA: Harvard Univ. Press, 20-46.

-------- (1961), Word and Object, Cambridge: MIT Press.

Ramsey, William (1992), "Prototypes and Conceptual Analysis,” Topoi 11: 59-70.

Reznek, Lawrie (1987), The Nature of Disease. London: Routledge \& Kegan Paul. 
Richman, Kenneth A. (2004), Ethics and the Metaphysics of Medicine: Reflections on Health and Beneficence. Cambridge, MA: MIT Press.

Rosch, Eleanor and Carolyn Mervis (1975), "Family Resemblance: Studies in the Internal Structure of Categories,” Cognitive Psychology 8: 382-439. Reprinted in DePaul and Ramsey (1998).

Rosenberg, Charles (1992), “Framing Disease: Illness, Society, and History.” In Framing Disease: Studies in Cultural History, eds. C.E. Rosenberg and J. Golden.

New Brunswick, NJ: Rutgers University Press. pp. xiii - xxvi.

Schwartz, Peter H. (2001), “Genetic Breakthroughs and the Limits of Medicine: Short Stature, Growth Hormone, and the Idea of Dysfunction,” St. Thomas Law Review 13 (4): 965-978.

-------- (2004), “An Alternative to Conceptual Analysis in the Function Debate,” The Monist 87 (1): 136-153.

Wakefield, Jerome C. (1992a), “The Concept of Mental Disorder: On the Boundary between Biological Facts and Social Values,” American Psychologist 47 (3): 373388.

-------- (1992b), “Disorder as Harmful Dysfunction: A Conceptual Critique of DSM-III-

R’s Definition of Mental Disorder,” Psychological Review 99 (2): 232-247. (1999a), “Evolutionary Versus Prototype Analyses of the Concept of Disorder,”

Journal of Abnormal Psychology 108 (3): 374-399

(1999b), “Mental Disorder as a Black Box Essentialist Concept,” Journal of

Abnormal Psychology 108 (3); 465-472.

Wittgenstein, Ludwig (1953), Philosophical Investigations. Trans. G.E.M. Anscombe.

New York: Macmillan Publishing Co., Inc. 


\section{Table 1: Definitions of Disease:}

King ([1954], 1981): "Disease is the aggregate of those conditions which, judged by the prevailing culture, are deemed painful, or disabling, and which, at the same time, deviate from either the statistical norm or from some idealized status." (1954, reprinted in Caplan et al., 1981, p. 112).

Culver and Gert (1982): "A person has a malady if and only if he has a condition, other than his rational beliefs and desires, such that he is suffering, or at increased risk of suffering, an evil (death, pain, disability, loss of freedom or opportunity, or loss of pleasure) in the absence of a distinct sustaining cause." (1982, p. 81)

\section{Boorse (1997):}

"1. The reference class is a natural class of organisms of uniform functional design; specifically, an age group of a sex of a species.

"2. A normal function of a part or process within members of the reference class is a statistically typical contribution by it to their individual survival and reproduction.

“3. A disease is a type of internal state which is either an impairment of normal functional ability, i.e. a reduction of one or more functional abilities below typical efficiency, or a limitation on functional ability caused by environmental agents.

“4. Health is the absence of disease. “ (pp. 7-8)

Wakefield (1992a, p. 384): “A condition is a disorder if and only if (a) the condition causes some harm or deprivation of benefit to the person as judged by the standards of the person's culture (the value criterion), and (b) the condition results from the inability of some internal mechanism to perform its natural function, wherein a natural function is an effect that is part of the evolutionary explanation of the existence and structure of the mechanism (the explanatory criterion).” 
Table 2: Classification of Theories:

\section{Dysfunction- \\ Requiring (DR) \\ Non-Dysfunction- \\ Requiring (non-DR)}

Value-

Requiring

Non-Value- $\quad$ Boorse (1997)

Requiring
King ([1954], 1981)

Culver and Gert (1982) 